\title{
Changes in Hospitalization Associated with Introducing the Resident Assessment Instrument
}

\author{
Vincent Mor, $\mathrm{PhD}$, Orna Intrator, $\mathrm{P} h D,{ }^{\dagger}$ Brant E. Fries, $\mathrm{P} h \mathrm{D}^{\ddagger}$ Charles Phillips, $\mathrm{PhD},{ }^{\mathrm{s}}$ \\ Joan Teno, MD, MS," Jeffrey Hiris, "Catherine Hawes, PhD, ${ }^{\text {a }}$ and John Morris, $P h D^{\|}$
}

OBJECTIVE: To compare the rates of hospitalization among cohorts of nursing home residents assembled before and after the implementation of the federally mandated Resident Assessment Instrument (RAI).

SAMPLE: Subjects were nursing home residents chosen from 268 facilities in major Metropolitan Statistics Areas in 10 states and representing more than 1500 facilities and 60,000 residents. Two resident cohorts (1990 and 1993) were sampled ( 8 to 16 residents per facility, depending upon facility size) as part of an evaluation of the impact of implementing the RAI.

METHODS: Research nurses reviewed records, interviewed staff, observed patients, and completed an RAI at baseline and 6 months later. All transitions during this interval (hospital admissions, nursing home transfers, returns home, death, etc.) were tracked. Using polytomous logistic regression, we tested the effect of cohort on the probability of being hospitalized in light of the competing risks of dying or remaining in the home, controlling for demographic and casemix variables, and having a DNR order in the chart.

RESULTS: A total of 4196 residents were studied, 2118 in 1990 (age 81.3 , female $77.7 \%$, LOS $6+$ months $49.8 \%$ ) and 2078 in 1993 (age 81.7, females $75.5 \%$, LOS 6+ months $50.2 \%$ ). The unadjusted probability of hospitalization dropped from .205 to .151 . Multivariate analyses revealed a significant adjusted odds of hospitalization of $.74(95 \% \mathrm{CI}$ $.60-.91)$ and no cohort effect on home discharge or death. Among severely cognitively impaired residents, the adjusted odds of hospitalization in 1993 compared with the 1990 cohort was $0.74(.53-1.03)$. Finally, among survivors in both cohorts who had a follow-up MDS performed, and whose ADL remained stable, $15.9 \%$ were hospitalized in 1990 , whereas only $10.9 \%$ were hospitalized in 1993 . On the other hand, ADL decliners were more likely to have been hospitalized in 1993 than in 1990 (40.6\% vs $25.2 \%)$.

\footnotetext{
From the "Center for Gerontology and Health Care Research, Brown University;

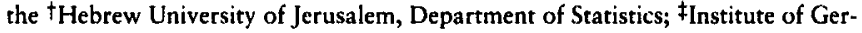
ontology and School of Public Health, University of Michigan and Ann Arbor

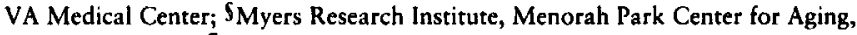
Beechwood, Ohio; George Washington Medical Center, Center to Improve Care of the Dying; and IInstitute for Research and Training, Hebrew Rehabilitation Center for Aged, Boston.

This paper was supported, in part, by HCFA Contract No. 500-88-0055 to the Research Triangle Institute.

Address correspondence to Vincent Mor, PhD, Center for Gerontology and Health Care Research, Brown University, Box G-B213, Providence, RI 02912.
}

CONCLUSIONS: Although other changes in the industry, clinical practice, and health care policy may have influenced hospitalization of nursing home residents, the substantial reductions observed among the cognitively impaired and those with stable ADL suggest superior and uniform assessment information in the form of the RAI contributed significantly to this decline. J Am Geriatr Soc 45:1002-1010, 1997.

$\mathrm{D}$ enson was among the first to recognize the dynamic pattern of the long term care careers of the frail elderly; he estimated that in 1986 nursing homes discharged $44 \%$ of residents admitted in that year to acute hospitals. ${ }^{1-6}$ Denson's simulations were derived from an inadequate information base, but represented the first effort to characterize movement among home, hospital and nursing home.

Changes in health care policies such as prospective hospital reimbursement and utilization review practices should influence the rates at which nursing home residents are hospitalized. However, little empirical data have been available to gauge the impact such policies have had on this rate. According to the 1985 National Nursing Home Survey, $49.1 \%$ of all live discharges from nursing homes were to an acute hospital, up from $41.1 \%$ in $1976 .^{7}$ There was also a higher rate of home discharge in $1985(37.2 \%)$ than in 1976 $(30.4 \%)$, consistent with the increasing use of nursing homes for post-acute care and rehabilitation. The proportion of all deaths occurring in nursing homes nationally also has increased dramatically during the 1980 's. ${ }^{8,9}$ That trend was greatest for the oldest old already living in nursing homes, only $31.8 \%$ of whom died in a hospital. ${ }^{10}$

\section{For editorial comment, see pp 975,1025 , and 1027}

Hospitalizing a nursing home resident is a major clinical issue known to entail increased risk of iatrogenisis and significant relocation stress. ${ }^{11}$ Indeed, many patients may be able to be treated in a familiar setting without increased mortality. ${ }^{12,13}$ Several studies examining nursing home patients' hospital admissions have found that between 7 and $50 \%$ could have been treated in the nursing home rather than being transferred to an acute hospital. ${ }^{14,1.5}$ Recently, clinical programs designed to increase the presence of physicians and nurse practitioners in nursing homes and expand the array of 
sub-acute services have been described, and all document substantial reductions in hospital use. One study estimated that $\$ 1$ billion could have been saved by treating residents in the nursing home. ${ }^{16}$

Two recent federal acts, the Nursing Home Reform Act embedded in OBRA (Omnibus Budget Reconciliation Act) ' 87 , which mandated the national implementation of the Resident Assessment Instrument (RAI) and the associated Minimum Data Set (MDS), and the Patient Self Determination Act (PSDA) of 1990 may have affected the rate at which nursing home residents are hospitalized. The RAI, through its comprehensive assessment, is designed to formulate holistic care plans that include consideration of residents' strengths, preferences, and social supports. The RAI uniformly focuses clinical assessor's attention on residents' physical and cognitive functioning, changes in functioning, the presence of various conditions that increase the rate of infection, pressure ulcers, dehydration, and other common geriatric syndromes. ${ }^{17}$ Because these factors may precipitate hospitalization, systematic documentation of their presence may help staff be more vigilant.

The PSDA implemented in 1991 mandated that patients be informed of their right to participate in treatment decisions and required institutions to document advanced directives. This may have led to increased doctor-patient (or doctor-family) discussions about the appropriateness of hospitalization. Indeed, the MDS provides a simplified format for recording the results of such discussions about advanced directives, indicating the presence of "Do Not Resuscitate Orders" and "Do Not Hospitalize" orders in the chart.

The purpose of this paper is to examine the impact of the RAI on the probability of hospitalization in a population of nursing home residents before and after the introduction of this policy. It is part of a larger evaluation of the impact of the RAI on the US nursing home industry and the residents it serves.

\section{METHODS}

This study used a quasi-experimental design in which four rounds of individual-level data collection were undertaken in 268 nursing homes in 1990 and 1993. Greater detail on many of the methodological issues associated with this study can be found elsewhere. ${ }^{18}$

\section{Sampling}

We purposely selected states for facility and resident sampling to assure that the RAI's impact would be evaluated in a very diverse set of policy environments. States were categorized in terms of geographic location (DHHS region), reimbursement system (case-mix or non-case-mix), Medicaid reimbursement level (low or high), and the average RN staffing patterns of a state's facilities. The states selected California, Ohio, Maryland, Connecticut, Minnesota, Oregon, Tennessee, Texas, Iowa, and Virginia - were relatively evenly arrayed across the various categories created by these variables.

Facilities were sampled from the counties within a single standard metropolitan statistical area (MSA). All nongovernmental, Medicare/Medicaid certified facilities with 25 or more beds constitute the sampling frame for the evaluation. We used a two-stage stratified design, with facilities (stratified by state and MSA or non-MSA status) serving as the first stage and residents within the home being the second stage. In the Spring of 1990 , a total of 317 eligible facilities (out of a universe of more than 500) were contacted by project staff and asked to participate in the study. Data were ultimately collected on residents in 268 facilities, comprising $85 \%$ of the eligible facilities contacted and chosen for participation by project staff. In the Spring of 1993, project staff recontacted these same 268 facilities, and 254 ( $80 \%$ of the eligible pool) participated in the post-RAI data collection.

In each participating facility, fixed target sample sizes of eight, 12 , or 16 residents (depending upon facility size) were selected from a resident census taken on the first day of data collection by research staff. Data were gathered on residents in the first cohort in 1990 and in the second cohort in 1993. Although 14 homes did not participate in the second wave of data collection, all resident level data from both waves were used in the analyses presented here.

\section{Data Collection}

All data necessary to complete an independently conducted MDS were gathered on each resident by research nurses with experience in geriatric nursing who had been trained by study investigators. The nurses spoke with and/or observed residents, reviewed records including up to 3 months of progress notes, and interviewed facility nursing staff and aides about each resident. The specific data collection protocols are described in detail elsewhere. ${ }^{19}$ The same protocol was used in 1990 and in 1993. Most data elements in the MDS have been shown to be highly reliable (based on inter-rater reliability) after training among both facility staff and research staff. ${ }^{19,20}$

Trained research nurses assembled data on sampled residents from three sources: records, talking with and/or observing the residents, and talking to caregiving staff such as aides and charge nurses. These three sources of data were integrated to best reflect the residents' functioning in each domain measured by the MDS. Research nurses were trained to consider that none of the sources alone was necessarily correct. Furthermore, consideration was given to the input of caregiving staff working on the evening and night shifts, because residents behavior or functioning might differ substantially. Since research staff spent an entire week in each facility, they were able to obtain the perspectives of multiple staff and observe sampled residents on numerous occasions.

Both the 1990 and 1993 resident cohorts were reassessed 6 months after the baseline data collection. For all residents, field staff noted the dates and sites of all transitions, including hospitalizations, transfers home or to other facilities, and mortality. Hospitalizations were defined as an overnight stay, not merely an emergency room visit. Residents no longer in the facility at the time of reassessment were tracked by a special telephone tracking unit with experience finding respondents in national panel studies. The unit recorded the dates and locations of all transitions (including death) occurring during the 6-month follow-up period, including when they were no longer able to reliably track the whereabouts of a sample member known to be alive (occurring for only 20 cases).

\section{Independent and Outcome Variables}

Independent variables were drawn from the baseline MDS assembled by research nurses in each cohort. The principal independent variable was cohort 1990 or 1993; it served as a proxy for the introduction of the MDS/RAI and 
the PSDA. Other independent variables introduced as controls in the analysis include a summary scale of dependence in the activities of daily living (ADLs), constructed as a hierarchy from selected $\mathrm{ADL}$ items in the $\mathrm{MDS},{ }^{21}$ the Cognitive Performance Scale ${ }^{\mathscr{O}}$ (CPS), which has been shown to replicate the Mini-Mental State Examination, ${ }^{22,23}$ the RUG-III Nursing Case Mix Index, ${ }^{24}$ age, gender, length of stay, and marital status. In view of the large increase in the rate of Do Not Resuscitate (DNR) orders in residents' charts between 1990 and $1993,{ }^{25}$ and to isolate the effect of cohort after controlling for this increase, we also used DNR as a covariate to control for the virtually simultaneous introduction of these policies. The presence of a Do Not Hospitalize Order was also examined, but it was quite rare in $1990(2 \%)$ and remained so in 1993 (4\%). There were very little missing data on any independent variable except marital status, our only measure of social support for which we adjusted missing values using mean substitution.

Two outcome variables were used for each patient: (1) the first transition event that occurred, defined as either hospitalization, death, discharge home, transfer to another facility, or no event occurred in the 6-month observation period (censoring) and (2) a count of the number of hospitalizations experienced. This latter measure is, of course, dependent on the likelihood of surviving the full 6 months of observation. Insufficient tracking data were present in 63 cases, resulting in an analytical sample of 4196.

\section{Analytical Approach}

Although data on all transitions patients experienced were available, most patients had only one event, and, thus, we chose to focus on the first event that occurred. A fourcategory outcome variable indicating "hospitalized," "discharged home," "died in nursing home," or "remained in a facility" (transfers to other facilities which accounted for less than $2 \%$ of all events were combined with those who remained in the facility) was analyzed using polytomous logistic regression. ${ }^{26}$ This procedure is a generalization of the more commonly used dichotomous logistic regression that may be used when there is an alternative outcome category that may occur instead of the event of interest (in this case hospitalization). The procedure yields one less equation than the number of outcome categories, that missing category constituting the referent group. In our analyses, the referent group is those residents remaining in a nursing home. The polytomous regression procedure generates regression coefficients for each parameter, that is, three different coefficients for each independent variable. Adjusted odds ratios with $95 \%$ confidence intervals were calculated for each coefficient in a manner identical to the procedure used for logistic regression.

In addition to the multivariate analyses of our categorical outcome variable, descriptive analyses of the probability and number of hospitalizations were performed, stratified by cohort, having a DNR and decedent status (dying within the 6-month observation period or not). To assess whether different patients were being hospitalized in 1990 and 1993, we also calculated a change in the ADL and/or CPS scales and compared the probability of hospitalization in 1990 and 1993 among those who improved, declined, or did not experience a two-point change in ADLs. A two-point change on these 6 and 7 level scales was found to be indicative of "permanent" change based on analyses of MDS data gathered quarterly from a different data set.

All analyses were weighted to reflect the population of residents in the $10 \mathrm{MSAs}$. Bivariate analyses and polytomous logistic regression were performed using Software for Survey Data Analyses (SUDAAN), with the unit of analysis being the individual resident. ${ }^{27}$ SUDAAN inflates the standard errors of estimate for simple proportions as well as for regression coefficients because our multi-stage sampling design results in observations within sampling cluster (facilities) being more alike than observations outside the cluster. The net result is a more accurate comparison of the differences between groups in the population (not in the sample).

\section{RESULTS}

The 4196 residents in the analysis sample experienced a total of 497 deaths, 1018 hospitalizations, 129 transfers to another nursing home, 129 discharges home, and 144 transfers to an "other" institution. Of these, 337 deaths occurred before a hospitalization or home discharge, 759 patients were hospitalized from the nursing home at least once, and 86 individuals were discharged home directly. The remaining deaths, hospitalizations, and home discharges occurred among patients first discharged home or to an "other" institution.

Table 1 describes the residents included in each cohort in terms of age, gender, marital status on admission, length of stay, cognitive and functional status, median RUG-III Nursing Case Mix Index (CMI), and presence of a DNR or Do Not Hospitalize order in the chart at baseline. There were no age differences between cohorts and only small, although statistically significant, differences in the distribution of the CPS scores. However, there is no consistent direction with regard to impairment level. The 1993 cohort appears to be significantly more impaired in ADLs in the mid range of the scale than is true for the 1990 cohort, which is consistent with the fact that the median RUG-III CMI was higher in the 1993 than in the 1990 cohort. Finally, we observe a virtual doubling in the rate of DNR orders between 1990 and 1993 and a similar rate of increase in DNH orders, although from only 2.6 to $4.4 \%$. Residents with DNH are a subset of those with DNR; all but seven persons with a DNH also had a DNR.

Table 2 presents the weighted raw percentage of all transitions from an originating state (e.g., original nursing home or a new nursing home to which the resident may have been transferred, a hospital or their home). Because some residents had multiple transitions of a given type (some were hospitalized up to four times), the percentages in the table represent the percent of transitions from one setting to another rather than the percent of residents who transited. The number at the top of the column reflects the estimated frequency of all transitions from that setting based on the weighted number of residents in our sample. The row labeled "Death" reflects the percentage of all transitions from a setting that ended in the death of the individual within the 6-months follow-up period. The final row represents the percent of transitions beginning in a given state who had no further transitions during the study period (in the first column this includes those who never left the original nursing home).

The first column, bottom row, reveals that most residents in either cohort were never discharged from the nursing home during the 6-month interval, although stability was more common in 1993 (65.9\% vs $72.3 \%$ ). The most com- 
Table 1. MDS Evaluation Resident Samples by Cohort

Percent of 1990 Cohort

$(n=2118)$
Percent of 1993 Cohort

$(n=2078)$

$\begin{array}{lrr}\text { Age } & & \\ <65 & 7.2 & 7.0 \\ 65-74 & 11.7 & 12.9 \\ 75-84 & 34.3 & 33.9 \\ 85-94 & 38.9 & 39.5 \\ 95+ & 8.0 & 6.7 \\ \text { Female } & 78.1 & 75.6 \\ \text { Married } & 47.7 & 46.7 \\ \text { Cognitive Performance Scale } & & \\ \text { Intact 1 } & 17.8 & 15.0 \\ 2 & 15.0 & 13.9 \\ 3 & 7.5 & 10.4 \\ 4 & 18.0 & 21.0 \\ 5 & 5.3 & 7.1 \\ 6 & 19.6 & 15.7 \\ \text { Impaired 7 } & 16.7 & 16.9 \\ \text { Activities of Daily Living Scale } & & \\ \text { Independent 1 } & 6.1 & 5.2 \\ 2 & 15.8 & 11.0 \\ 3 & 14.6 & 16.6 \\ 4 & 11.8 & 15.9 \\ 5 & 30.2 & 30.4 \\ \text { Totally dependent 6 } & 21.8 & 20.9 \\ \text { Median RUGs III Nursing Case Mix } & .96 & 1.02 \\ \text { Index } & & \\ \text { Has a Do Not Resuscitate order } & 31.0 & 51.4 \\ \text { Has a Do Not Hospitalize order } & 2.6 & 4.4 \\ \text { Total deaths (during 6 month } & 11.4 & 10.8 \\ \text { observations) } & & \end{array}$

Table 2. Percent of Transition from One Setting to Each Destination in Six Months by Cohort (Weighted No. Represents 190, 161 Transitions)

\begin{tabular}{|c|c|c|c|c|c|c|c|c|}
\hline \multicolumn{9}{|c|}{ Transition from: } \\
\hline \multirow[t]{3}{*}{ Cohort } & \multicolumn{2}{|c|}{ Original $\mathrm{NH}$} & \multicolumn{2}{|c|}{ New NH } & \multicolumn{2}{|c|}{ Hospital } & \multicolumn{2}{|c|}{ Home } \\
\hline & \multicolumn{2}{|c|}{ Cohort } & \multicolumn{2}{|c|}{ Cohort } & \multicolumn{2}{|c|}{ Cohort } & \multicolumn{2}{|c|}{ Cohort } \\
\hline & $\begin{array}{l}1990 \\
\text { No. T }\end{array}$ & $\begin{array}{l}1993 \\
\text { itions }\end{array}$ & No. Transitions & $\begin{array}{l}1993 \\
\text { sitions }\end{array}$ & No. Transitions & $\begin{array}{l}1993 \\
\text { sitions }\end{array}$ & $\begin{array}{c}1990 \\
\text { No. } T\end{array}$ & $\begin{array}{l}1993 \\
\text { sitions }\end{array}$ \\
\hline Transition to & 76050 & 67106 & 1538 & 1249 & 16777 & 11071 & 1924 & 929 \\
\hline Original NH & & & $4.0 \%$ & $4.1 \%$ & $74.9 \%$ & $76.9 \%$ & $32.5 \%$ & $1.2 \%$ \\
\hline New NH & 1.3 & 1.5 & & & 2.6 & 2.0 & 4.0 & .3 \\
\hline Hospital & 21.0 & 16.0 & 6.9 & 13.4 & & & 26.0 & 15.7 \\
\hline Home & 1.9 & 1.1 & 5.1 & 0.0 & 2.3 & 1.3 & & \\
\hline Death & 6.8 & 7.5 & 3.9 & 3.6 & 10.2 & 10.8 & 2.0 & 0.0 \\
\hline No transition from this state & 65.9 & 72.3 & 79.8 & 77.9 & 9.4 & 9.8 & 35.3 & 81.1 \\
\hline
\end{tabular}

mon transition from the original nursing home was to hospital, and a large difference between cohorts is obvious, $21 \%$ versus $16 \%$. Only a small proportion of transitions were to another nursing home $(1.3 \%$ and $1.5 \%)$. The rate of home discharge from the original facility was marginally higher in 1990 than in $1993(1.9 \%$ and $1.1 \%)$. Although the overall death rate remained constant $(8.2 \%$ vs $8.4 \%)$ the rate of nursing home deaths was marginally higher in 1993 (6.8\% vs $7.5 \%)$.

Most of the small number of individuals transferred to another nursing home (just under $80 \%$ ) experienced no further transitions. Among the many hospitalizations, about 
three-quarters $(74.9 \%$ vs $76.9 \%)$ returned to the original nursing home. About $10 \%$ of hospitalizations of nursing home residents ended in death. Finally, the relatively small number of discharges home appear to have had a volatile experience. For example, among those who went home, more subsequent transitions were readmissions to the original or to a new nursing home in $1990(32.5 \%+4.0 \%)$ than in 1993 $(1.2 \%+.3 \%)$. However, all these figures are based upon a small number of unweighted individuals and transitions.

Table 3 presents the distribution of the first events experienced by residents in the two cohorts. In $1990,20.5 \%$ of residents were hospitalized directly from the nursing home at least once, compared with only $15.1 \%$ of those in the 1993 cohort. Only $1.3 \%$ of residents in each cohort were discharged home, and $7.1 \%$ and $7.6 \%$ of each cohort were discharged dead. These resident-level findings largely parallel the transition data presented in Table 2.

Because some residents experienced multiple hospitalizations, we examined the distribution of hospitalizations by cohort separately for those residents who did and did not survive the entire 6 -month observation period. Table 4 presents the results of these analyses, revealing that both survivors and decedents were more likely to have been hospitalized both once and more than once in 1990 than in 1993.

\section{Polytomous Regression Results}

Table 5 presents the results of the categorical logistic regression analyses. For each independent variable in the model, an adjusted odds ratio and associated $95 \%$ confidence interval are presented for each of three outcome states. The odds ratios stipulate the odds of the event occurring relative to not occurring (remaining in the facility), all conditioned on not having experienced one of the other two events.

Thus, the odds of being discharged to hospital in the 1993 cohort are $.74(95 \%$ CI .60 - .91) of those for the 1990 cohort, conditioned on surviving and not being discharged home and controlling for various resident mix factors such as the ADL Scale, CPS, length of stay and RUG-III CMI, as well as being female, married or having a DNR in the record. As can be seen, DNR has an independent effect on the risk of hospitalization (AOR .63;.51 - .79). Placement in the most impaired half of the Cognitive Performance Scale distribution also reduces the risk of hospitalization (AOR .94; .88-.99) significantly. Female residents are significantly less likely to be hospitalized (AOR .65, .52 - .83), whereas, those who are married are significantly more likely to be admitted to hospital (AOR 1.49; $1.04-2.15$ ). Age, CMI, and ADL at baseline were unrelated to the risk of hospitalization.
Table 4. Numbers of Hospital Admissions Among Survivors and Decedents by Cohort (Weighted Using SUDAAN)

\begin{tabular}{lccccc}
\hline \multirow{2}{*}{$\begin{array}{c}\text { Number of Hospital } \\
\text { Admissions }\end{array}$} & \multicolumn{2}{c}{$\begin{array}{c}\text { Survivors } \\
\text { Cohort }\end{array}$} & & \multicolumn{2}{c}{$\begin{array}{c}\text { Decendents } \\
\text { Cohort }\end{array}$} \\
\cline { 2 - 3 } \cline { 5 - 6 } None & 1990 & 1993 & & 1990 & 1993 \\
1 & $80.7 \%$ & $85.9 \%$ & $62.9 \%$ & $71.9 \%$ \\
$2+$ & 15.5 & 12.0 & & 27.6 & 20.9 \\
\cline { 1 - 2 } & 3.9 & 2.2 & & 9.4 & 7.2 \\
\hline
\end{tabular}

* Weighted SUDAAN Analyses; see page 10 in Methods.

No significant cohort effects were observed in the polytomous regression model for either the probability of discharge home or death. Those with DNR orders were almost twice as likely to die (AOR 1.97; 1.50-2.58), and those in the poor ADL group had a $40 \%$ increased risk of death (AOR $1.40 ; 1.18-1.66$ ). Also, women were significantly less likely to die, and the oldest residents had a higher risk of death. Finally, the higher the CMI, the greater the likelihood of death.

In view of the large cohort effect on hospitalization and the importance of severe cognitive function in reducing hospital risk without increasing mortality, we ran a polytomous regression model adding a term representing the interaction of poor cognition and cohort. Controlling for all the factors in the model in Table 5, including the main effects of cohort and cognitive function, severely cognitively impaired residents in 1993 were less likely (AOR .74; .53 - 1.03) to have been hospitalized than their counterparts in 1990 .

Figure 1 displays this relatively large reduction in hospital use for those in 1993 who were cognitively impaired. In $1990,20.1 \%$ of those with severely impaired cognition were hospitalized at least once, in contrast to $21.1 \%$ of those not so impaired. In 1993, these percentages dropped to $13.5 \%$ and $17.7 \%$. Thus, there is both a clear main effect of cohort as well as an interaction between poor CPS and being in the 1993 cohort.

To explore further the hypothesis that the MDS helped target patients for hospitalization more appropriately, we looked at changes in ADL and cognitive function among survivors to see if hospital use differed by cohort within strata defined by change in function. Considering only changes in CPS of at least 2 levels, most survivors (about two-thirds) evidenced no change. In 1990, $15.2 \%$ of those who didn't change were hospitalized, whereas only $10.1 \%$ of those with

Table 3. Percentage of Nursing Home Residents in 1990 and 1993 Cohorts Discharged from Nursing Home to Hospital, Home, or Who Were Dead on Discharge

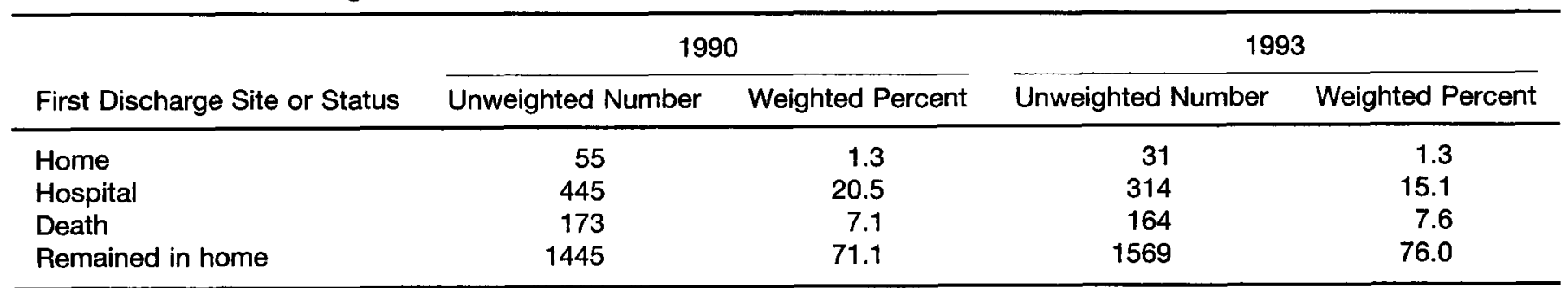

* Weighted SUDAAN Analyses; see page 10 in Methods. 
Table 5. Polytomous Logistics Regression Results of Predictors of First Discharge Site Relative to Remaining in the Facility. Adjusted Odds Ratios and $95 \%$ Confidence Intervals

\begin{tabular}{|c|c|c|c|}
\hline \multirow[b]{2}{*}{ Individual Variables } & \multicolumn{3}{|c|}{ First Discharge Site } \\
\hline & Home & Hospital & Death \\
\hline $\begin{array}{l}\text { Cohort } \\
(93 \text { vs. } 90)\end{array}$ & $\begin{array}{l}0.98 \\
55175)\end{array}$ & $0.74^{\star \star \star}$ & 0.83 \\
\hline $\begin{array}{l}\text { (93 vs. } 90) \\
\text { Cognitive Performance Scale }\end{array}$ & $\begin{array}{c}(0.55,1.75) \\
0.61^{\star \star \star}\end{array}$ & $\begin{array}{c}(0.60,0.91) \\
0.94^{\star \star}\end{array}$ & $\begin{array}{l}(0.63,1.11) \\
0.97\end{array}$ \\
\hline (Poor vs. OK) $^{+}$ & $(0.53,0.71)$ & $(0.88,0.99)$ & $(0.89,1.06)$ \\
\hline Do Not Resuscitate order & 0.99 & $0.63^{\star \star \star}$ & $1.97^{\star \star \star}$ \\
\hline (Yes vs. No) & $(0.53,1.81)$ & $(0.51,0.79)$ & $(1.50,2.58)$ \\
\hline ADL level & $0.81^{*}$ & 1.07 & $1.40^{\star \star \star}$ \\
\hline (Poor vs. OK) $^{++}$ & $(0.64,1.03)$ & $(0.97,1.18)$ & $(1.18,1.66)$ \\
\hline \multirow[t]{2}{*}{ Female } & 0.83 & $0.65^{\star \star \star}$ & $0.65^{\star \star \star}$ \\
\hline & $(0.45,1.52)$ & $(0.52,0.83)$ & $(0.46,0.91)$ \\
\hline \multirow[t]{2}{*}{ Married } & $10.37^{\star \star \star}$ & $1.49^{\star \star}$ & $1.58^{*}$ \\
\hline & $(4.70,0.23)$ & $(1.04,2.15)$ & $(0.93,2.67)$ \\
\hline \multirow{2}{*}{$\begin{array}{l}\text { Long stay resident (60 } \\
\text { days }+ \text { ) }\end{array}$} & 0.71 & 0.97 & 1.21 \\
\hline & $(0.39,1.30)$ & $(0.78,1.19)$ & $(0.92,1.59)$ \\
\hline \multirow[t]{2}{*}{ Age (log form) } & $0.30^{* *}$ & 1.03 & $13.20^{* * *}$ \\
\hline & $(0.10,0.93)$ & $(0.60,1.76)$ & $(3.11,56.16)$ \\
\hline \multirow[t]{2}{*}{ Nursing case mix index } & $3.82^{\star \star \star}$ & 1.32 & $2.21^{* \star \star}$ \\
\hline & $(2.01,7.25)$ & $(0.90,1.95)$ & $(1.51,3.23)$ \\
\hline
\end{tabular}

$* * * P<.001$

$* * P<.05$

$* P<.01$

+ "OK" on Cognitive Performance Scale means NOT being in 3 severely impaired levels of 7-point scale.

++ "OK" on ADL. Level means NOT being fully dependent in eating and at least 2 other ADLs.

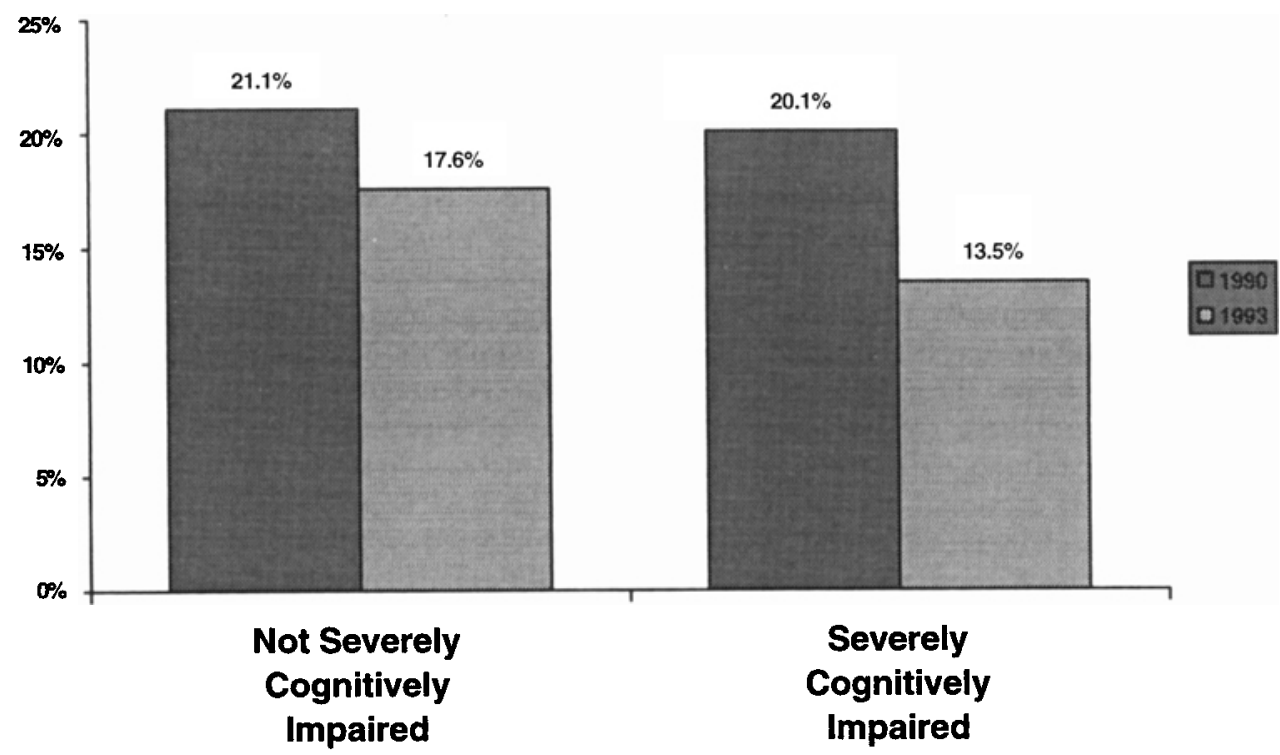

Figure 1. Effect of cohort and cognitive impairment on 6-month hospitalization rates.

relatively stable cognition were hospitalized in 1993 . On the other hand, the hospital use rate of survivors who declined was similar in the two cohorts $(20.6 \%$ vs. $21.2 \%)$, as was the case for the minority who improved $(16.1 \%$ vs. $14.5 \%)$. Applying the same rules to change in ADL, we observed the same phenomenon of reduced hospital use among those with stable ADL (15.9\% in 1990 vs $10.9 \%$ in 1993) but actually increased hospital use among survivors who declined in ADL function $(25.2 \%$ vs $40.6 \%)$.

\section{DISCUSSION}

We examined the rates and destinations of discharges from nursing homes in two cohorts assembled from the same facilities in 1990 and in 1993 to explore the impact of the introduction of the Congressionally mandated Resident Assessment Instrument. We observed a $28 \%$ reduction in the 6 -month rate of hospitalization with no increase either in mortality, or in the likelihood of home discharge. Although the concurrent introduction of the Patient Self-Determination 
Act complicates any interpretation of our findings, by simultaneously examining the effect of increases in the prevalence of DNR orders, our results suggest that hospitalizations were less prevalent in 1993 even after adjusting for the 60\% increase in the prevalence of DNR orders. Among the most cognitively impaired residents, hospital use rate dropped from 20.1 to $13.5 \%$, and this effect was independent of the effect of having a DNR on hospital use. Furthermore, exploratory analyses of changes in hospital use among survivors whose ADL or cognition did or did not change over the 6-month observation period reveal that the biggest reductions in hospital use occurred among those whose functioning remained fairly stable. Finally, the likelihood of hospitalization fell from 1990 to 1993 more rapidly among decedents than amongst survivors.

These results suggest that the RAI made it possible to target better hospital use, reducing admissions among those with the least likelihood of benefiting from them: the severely cognitively impaired, the terminally ill, and those who may not need them. This reduction in hospitalization occurred with only a small reduction in hospitalization of those without serious cognitive impairment and no reductions among survivors whose function declined or improved.

We recognize that there are many other factors that may have contributed to changes in the use of hospitals among nursing home residents, including the changing role of nursing homes as specialty care, sub-acute and terminal care facilities. ${ }^{28}$ However, evidence from this evaluation, presented elsewhere, suggests that introducing the RAI resulted in substantial changes in organizational practices and in the processes of care used in a large proportion of nursing homes. ${ }^{18,29}$ These findings speak to the viability of our study hypothesis. Since both the MDS and the PSDA may have had direct and indirect effects on hospital use, disentangling the effect of both of these policy changes is necessarily complex. The paragraphs below consider several alternate explanations for our results and conclude that in all likelihood, the introduction of the RAI contributed to the observed reduction in hospital use among nursing home residents.

The first question to raise is whether the observed reduction in the rate of hospital use of nursing home residents is merely a reflection of a secular trend. Information on this issue is not readily available, and there are no nationally representative series of data pertaining to hospital use of nursing home residents. The 1985 National Nursing Home Survey found that $35.1 \%$ of all discharges in that year were to an acute hospital ( $49.1 \%$ of live discharges), which is consistent with Denson's estimate of between $30 \%$ and $45 \%{ }^{1}$ Between 1990 and 1993, there were no policy changes that occurred in the acute hospital sector likely to have been responsible for the observed change. More aggressive preadmission certification programs were not applied to the Medicare beneficiaries who made up almost all of our population, and no states in which our study was conducted were undertaking a major effort to reduce hospital admissions of nursing home residents. Indeed, an examination of the acute hospital discharge rates per 1000 patients aged $65+$ between 1990 and 1992 reveals an increase (327.1 to 336.5), rather than a decrease. This suggests that a reduction in hospital use by the nursing home population was contrary to the national trend. ${ }^{30-32}$ Unless there was a substantial reduction in these rates in the latter part of the 1980s in ways not reported in the literature, it is likely that the policy interventions introduced between 1990 and 1993 were responsible for the substantial drop reported here.

One possible trend noted in the literature that could explain some of our findings is the increasing use of nursing homes as a place to die. ${ }^{8-10}$ Whereas our data reveal an increase between 1990 and 1993 in the proportion of deaths that occurred in the nursing home $(73.8 \%$ vs $80.0 \%)$, the size of this difference pales by comparison with the drop in hospital use from $20.5 \%$ to $15.1 \%$. Although a disproportionate share of the reduction in hospital use is attributable to decedents $(37.1 \%-28.1 \%)$, this does not account for even half of the observed difference.

Parceling out the relative contributions of the RAI or the PSDA to reducing hospital use requires that we consider the intended consequences of each policy. The PSDA mandated that healthcare facilities ask patients if they have made advanced directives and to facilitate discussion. ${ }^{25}$ Several studies of the implementation of the PSDA in the hospital environment conclude that the law did not achieve its intended goals. ${ }^{33}$ We observed an increase from $31 \%$ of residents having a DNR documented in the chart in 1990 to more than $50 \%$ of residents having a DNR documented in 1993. An advance directive specifying DNH was quite rare in both 1990 and 1993 ( $2 \%$ and $4 \%$, respectively), suggesting that reductions in hospital use came about by a misapplication of DNR orders rather than by a considered, informed discussion of treatment alternatives in the event of a clinical situation that might call for hospitalization. Had the PSDA had its intended effect, we should have observed an absolute increase in DNH orders commensurate with the reduction in the hospitalization rate; we saw no such increase.

Another secular trend over the past several years that might explain reduced hospital use is the emergence of subacute services in U.S. nursing homes. ${ }^{28}$ While this is known to be concentrated in certain types of markets and in selected types of facilities, it may contribute to the observed reduction in hospital use. Comparing our sample facilities in 1990 and 1993, we found no difference in either staffing intensity or case mix (data not shown). Even the proportion of facilities with on-staff medical directors did not change. While there was a significant increase in the acuity and medical complexity of residents in these facilities, this should have been associated with an increase in hospital use unless there was a larger increase in staffing levels and sophistication.

The RAI is a clinical assessment system designed for individualized careplanning. Interviews of nursing leadership in facilities participating in our study suggest that while there may have been complaints about how it was implemented and the perceived paperwork burden, the clinical utility of the RAI was widely appreciated, with some staff noting that they were able to uncover clinical problems that they would otherwise have missed. To the extent that hospitalizations of nursing home residents occur because of acute exacerbations of chronic conditions and are caused by complications from avoidable care problems (infections, pressure ulcers, falls, etc.), it is logical to suspect that, in the long run, the RAI may reduce hospital admissions. It is remarkable that after merely 2 years, we were able to observe a reduction in a health service utilization indicator that is normally quite insensitive to all but the most pervasive clinical interventions.

To place the size of the observed effect in context with effects other policy interventions have had on hospitalization rates, let us consider the Medicare Catastrophic Coverage 
Act (MCCA). The 1-year (1989) it was in effect, Medicare eligible nursing home residents who became clinically eligible for Medicare skilled nursing services could receive them without first having to pass through an acute hospital. Analyses of the hospitalization rate among a national sample of Medicare eligible nursing home residents revealed only a small reduction in the rate of hospitalization between 1988 and 1989 , controlling for episode duration. ${ }^{34}$

Variation in state policies and practices may contribute to variation in the observed hospitalization rate; for example, 1990 rates of 14.5 in Oregon and 34.4 in Texas and Ohio. However, in spite of state differences in regulatory, reimbursement, and bed supply policies, reduction in hospital use was observed in all 10 study sites. It is likely that facility factors, particularly the availability of clinical resources, may influence the propensity to hospitalize residents. Indeed, Teresi and her colleagues found that nursing homes varied tremendously in their propensity to hospitalize residents: facilities with inadequate nursing coverage and those with fewer diagnostic resources hospitalized them more frequently. ${ }^{35}$ A recently published population-based study from Rochester in the 1980s also found hospitalization rates were lower in nursing homes with on-site medical staffs. ${ }^{36}$ Future research is needed to simultaneously estimate the effect of policies, market factors, and facility resources on the likelihood of hospitalization, with an eye both to designing policies to reduce inappropriate hospitalizations and to stimulating nursing facilities to develop the resources and/or relationships with medical groups to meet patients' medical needs without having to admit them to a hospital.

Beyond their substantial policy implications, these findings add considerably to our knowledge of the longitudinal experience of nursing home residents. Our study is the first using a nationally representative probability sample of nursing home residents to document the high degree of flux that characterizes the experience of individuals leaving the nursing home, if only temporarily. The findings are reminiscent of the multi-facility study undertaken by Lewis and her colleagues in southern California. ${ }^{3}$ While the majority of hospitalizations return to the originating nursing home, more than $10 \%$ end in death, and some result in a transfer to another facility. We found little evidence for the phenomenon of nursing homes hospitalizing patients as a means of transferring them to another setting. This does not mean that this doesn't occur; rather it is not prevalent and may be concentrated in some facilities. This will have to be explored in future studies.

In closing, we feel that it is reasonable to conclude that the introduction of the MDS contributed to a reduction in the hospitalization rate among nursing home residents. Although any interpretation of the effects of history are fraught with difficulty, we believe that the observed rate of decline is both sufficiently large and independent of the large increase in the use of Do Not Resuscitate orders that no other explanation is sufficient. Unfortunately, as with most policy evaluations, the hard evidence of a randomized clinical trial will always be lacking. Nonetheless, when considered in conjunction with very substantial changes in the processes of nursing home care that also accompanied the introduction of the MDS, the notion that better assessments contributed to a reduction in hospitalizations is not implausible.

\section{REFERENCES}

1. Densen PM. The elderly and the health care system: Another perspective, Milbank Q 1987;6.5:614-6.38.

2. Kane R, Kane R. Long Term Care. Principles Programs and Policies., New York: Springer Publishing Company, 1987.

3. Lewis MA, Cretin S, Kane RL. The natural history of nursing home patients. Gerontologist 1985;25:382-388.

4. Lewis MA, Lcake B, leal-Sotelo M, Clark V. First nursing home admissions: Time spent at home and in instirutions after discharge. Am J Public Health 1990;80:22-24

5. National Center for Health Statistics. Health United States. Publication No. (I'HS): 86-1232. Washington, DC, 1985.

6. Meiners MR. The state of the art of long term care insurance. In: Long-Term Care Financing and Delivery Systems: Exploring Some Alternatives. Conference procecdings. HCFA I'ublication No. 03174. Washington, DC: I lealth Care Financing Administration, 1984.

7. Sekscenski FS. Discharges from nursing homes. P'reliminary data from the 1985 National Nursing Home Survey. Advance Data from Vital and Health Statistics. National Center for Health Statistics, No. 142. DHHS Publications No. (PHS)87-12.50. Hyattsvillc, MD: Public Health Service, 1987.

8. McMillian A, Mentnech R, L.ubitz J et al. Trends and patterns in place of death for Medicare enrollecs. Health (are Financ Rev 1990; 12:1-7.

9. Sager MA, Easterling DV, Kindig DA, Anderson OW. Changes in the location of death after passage of Medicare's prospective payment system: A national study. N Engl J Med 1989;320:4.3.3-439.

10. Merrill D, Mor V. l'athways to hospital death among the oldest old. J Aging Health 1993;5:516-535.

11. Gillick M, Stcel K. Referral from long-term care to acute facilitics. J Am Geriatr Soc 1993;31:74-78.

12. Bergman H, Clarfield AM. Appropriatencss of patient transfer from a nursing home to an acute-care hospital: $A$ study of emergency room visits and hospital admission. J Am Geriatri Soc 1991;39:1164-1168.

13. Kerr HD, Byrd JC. Nursing home patients transferred by ambulance to a VA emergency department. J Am Geriatr Soc 1991;39:132-136.

14. Wofford JL, Schwartz E, Byrum JE. The role of emergency services in health care for the elderly: A revicw. J Emerg Med 1993;11:317-326.

15. Zimmer JG, Eggert CM, Truat A, Brodows B. Nursing homes as acute care providers: A pilot study of incentives to reduce hospitalizations. J Am Geriatr Soc $1988 ; 36: 124-129$

16. Kayser-Jones JS, Weiner Cl, Barbaccia JC. Factors contributing to the hospitalization of nursing home residents. Cerontologist 1989;29:502-510.

17. Zweibel NR, Cassel C.K, eds. Clinical and policy issues in the carc of the nursing home patient. (linics in Geriatric Medicine. Philadelphia: W.B. Saunders, 1988.

18. Phillips C, Morris JN, Hawes Cet al. Assosciation of the Resident Assessment Instrument (RAl) with changes in function, cognition, and psychosocial status. J Am Geriatr Soc 1997;45:986-993.

19. Morris JN, Hawes C, Fries BE et al. Designing the National Resident Assessment for nursing homes. Gerontologist 1990;30:293-307.

20. Hawes $C$, Morris JN, Phillips CD. Reliability estimates for the Minimum Data Set for nursing home resident assessment and care screening (MDS). Gerontologist 1995;35:172-178.

21. Mor V, Branco K, Fleishman J et al. 'The structure of social engagement among nursing home residents. J Cierontol: Psychol Sci 1995;50:P1-8.

22. Morris JN, Fries BF, Mchr DR et al. MDS Cognitive Performance Scale. ] Gerontol: Med Sci 1994;49:M174-182.

23. Hartmaier S, Sloane P, Guess H, Roch C. The MDS Cognition Scale. A valid instrument for identifying and staging nursing home residents with dementia using the MDS. J Am Cieriatr Soc 1994;42:1173-1179.

24. Fries BF, Schneider DP, Foley WJ et al. Refining a case-mix measurc for nursing homes: Resource utilization groups (RUGs-III). Med Care $1994 ; 32 ; 668-68.5$.

25. Teno J, Branco $\mathrm{K}$, Mor $\mathrm{V}$ et al. Changes in advance care planning in nursing homes before and after the Patient Self-Determination Act: Report of a 10 state survey. J Am Geriatr Soc 1997; 45:939-944.

26. Hosmer DW, I emeshow S. Applied Logistic Regression. New York: John Wiley \& Sons, 1989.

27. Research Triangle Institute. Software for Survey Data Analysis (SUDAAN), Version 5.30 for DEC: Vax Mainframes and Workstations (VMS) and for IBM Personal Computers and Compatibles (MS DOS). Rescarch Triangle Park, NC: Research Triangle Institute, 1989.

28. Zinn J, Mor V. Nursing home special care units: Distribution by type, state and facility characteristics. Gerontologist 1994;34:371-377.

29. Hawes $C$, Mor V, Phillips $C D$ et al. The OBRA-87 nursing home regulations and implementation of the Resident Assessment Instrument: Effects on process quality. J Am Geriatr Soc 1997;45:977-98.5. 
30. Graves EJ. Detailed diagnoses and procedures, National Hospital Discharge Survey, 1992. National Center for Health Statistics, Vital Health Statistics, Series 13, No. $118,1994$.

31. Graves EJ. Detailed diagnoses and procedures, National Hospital Discharge Survey, 1991. National Center for Health Statistics, Vital Health Statistics, Series 13, No. 115, 1994.

32. Graves EJ. Detailed diagnoses and procedures, National Hospital Discharge Survey, 1990. National Center for Health Statistics, Vital Health Statistics, Series 13, No. 113, 1992.
33. Hastings Center Report Special Supplement November-December, 1994.

34. Laliberte L, Mar V, Berg K et al. Impact of the Medicare Catastrophic Coverage Act on nursing homes. Milbank Q 1997;75:1-31.

35. Teresi J, Holmes D, Bloom HG et al. Factors differentiating hospital transfers from long term-care facilities with high and low transfer rates. Gerontologist 1991;31:795-806.

36. Barker WH, Zimmer JG, Hall WJ et al. Rates, patterns, causes and costs of hospitalization in nursing home residents: A population-based study. Am J Public Health 1994;84:1615-1620. 